\title{
Rendimiento productivo e hígado graso en tilapia híbrida (Oreochromis spp): Influencia de dos fuentes de lípidos
}

\author{
Productive performance and fatty liver in hybrids of red tilapia \\ (Oreochromis spp): Effects of two dietary lipid sources
}

\section{Desempenho produtivo e fígado gordo em híbridos de tilápia (Oreochromis spp): Influência de duas fontes lipídicas na dieta}

\author{
Pedro A. Triana-García ${ }^{*}$, Mariana C. Gutierrez-Espinosa ${ }^{2}$, Pedro R. Eslava-Mocha ${ }^{3 *}$. \\ MVZ. \\ Zootecnista, MSc, Grupo de Investigación Instituto de Acuicultura Universidad de los Llanos \\ MV, MSc. \\ Grupo de investigación en sanidad de organismos acuáticos- Instituto de acuicultura de la Universidad de los Llanos \\ Email:alejotriana11@hotmail.com
}

Recibido: Mayo 14 de $2013 \quad$ Aceptado: Octubre 25 de 2013.

\begin{abstract}
Resumen
Con el objeto de evaluar el efecto de dos fuentes de lípidos de origen animal y vegetal en el rendimiento productivo y la presentación de hígado graso en alevinos de tilapia híbrida (Oreochromis spp), se utilizaron seis dietas con aceite de pescado y aceite de soya como fuente de lípidos, con niveles de inclusión del 5\%, 9\% y 13\% de cada uno, durante un periodo de 60 días. Se determinaron parámetros de rendimiento productivo e índices corporales (índice hepatosomático-IHS, índice viscerosomático-IVS). Adicionalmente se realizó evaluación histopatológica del hígado con tinciones diferenciales para lípidos y carbohidratos. Se observó un aumento significativo de peso $(p<0.05)$ en los tratamientos con niveles de inclusión del 5\%, 9\% y 13\% de aceite de soya respecto de los tratamientos con aceite de pescado con niveles de inclusión del $9 \%$ y $13 \%$. Con la fuente de lípidos de origen vegetal se observó disminución significativa $(p<0.05)$ en el IHS para el tratamiento con un nivel de inclusión del 13\%, con respecto a los tratamientos con un nivel de inclusión del 5 y $9 \%$ de la fuente de lípidos de origen animal. El IVS no presentó diferencias significativas entre tratamientos. La severidad de la infiltración grasa en el hígado fue menor en los tratamientos del 5\%, 9\% y $13 \%$ de aceite de soya. De acuerdo a los resultados observados, puede inferirse que el aceite de soya, rico en ácidos grasos poliinsaturados n-6, mejora el rendimiento productivo y disminuye la incidencia de lipidosis hepática en las tilapias híbridas en comparación con el aceite de pescado, fuente de lípidos ricas en ácidos grasos n-3 y poliinsaturados de cadena larga.
\end{abstract}

Palabras clave: Lipidosis hepática, Tilapia, Ácidos grasos, metabolismo de lípidos.

\begin{abstract}
With the aim to evaluate the effect of animal and vegetable lipids on productive performance and development of fatty liver in fingerlings of hybrid tilapia (Oreochromis spp), six diets whit fish oil and soy bean oil as
\end{abstract}


source of lipids were performed. The lipid levels were 5, 9 and $13 \%$ of each source, and the feeding period was 60 days. Values of productive performance parameters, hepatosomatic index-HIS and viscerosomatic index-IVS were determined. Additionally, liver histopathologic evaluation was performed with differential stains for lipids and carbohydrates. Weight increase of fish fed with 5, 9 and $13 \%$ of soy bean oil was observed as compared with those fed with 5 and $9 \%$ of fish oil. Soy bean oil at $13 \%$ in diets reduced the HSI of fish as compared with those fed with 5 and $9 \%$. No differences were observed among treatments concerning SVI. The severity of fat infiltration was lower in fish fed diets with 5, 9 and $13 \%$ of soy bean oil. The present set of data allows to inferring that dietary soy bean oil, a n-6 polyunsaturated fatty acids rich nutrient, improves the productive performance and decreases the incidence of hepatic lipidosis of hybrid tilapias as compared with fish oil, a n-3 polyunsaturated fatty acids and long chain polyunsaturated fatty acids rich nutrient.

Keywords: hepatic lipidosis, Tilapia, Free fatty acids, lipid metabolism.

\section{Resumo}

Com fins de avaliar o efeito de duas fontes lipídicas, uma de origem animal e outra vegetal, sobre o desempenho produtivo e o desenvolvimento de fígado gordo em alevinos de tilapia híbrida (Oreochromis spp), foram utilizadas seis dietas com óleo de peixe e óleo de soja como fonte de lipídeos. Os níveis de inclusão foram 5, 9 e 13\% de cada um, e a alimentação ocorreu em um período de 60 dias. Foram determinados os parâmetros de rendimento produtivo, índices corporais (índice hepatossomático-IHS, índice viscerosomático índice-IVS). A avaliação histopatológica do fígado foi realizada utilizando-se colorações diferenciais para lipídios e carboidratos. Observou-se um aumento de peso significativo $(p<0,05)$ nos peixes tratados com níveis de inclusão de 5, 9 e 13\% de óleo de soja na dieta em relação aos tratamentos com inclusão de 9 e 13\% de óleo de peixe. Os peixes alimentados com 13\% de óleo de soja apresentaram decréscimo significativo ( $p$ $<0,05)$ no IHS quando comparados com peixes tratados com níveis de inclusão de 5 a $9 \%$ de farinha de peixe; o IVS não foi diferente entre os tratamentos. A gravidade da infiltração de gordura no fígado foi menor para o tratamento com 5, 9 e 13\% de óleo de soja, em comparação com os tratamentos contendo 5, 9 e 13\% de óleo de peixe. Do conjunto de dados apresentados, pode-se inferir que o óleo de soja, rico em ácidos graxos poli-insaturados n-6, aumenta a produtividade e reduz a incidência de lipídios no fígado de tilápias híbridas quando comparado ao óleo de peixe, uma fonte rica em ácidos graxos n-3 e poli-insaturados de cadeia longa.

Palavras chave: Fígado gordo, Tilápia, ácidos graxos, metabolismo lipídico

\section{Introducción}

Estimar los requerimientos nutricionales de las especies cultivadas con el propósito de mejorar su rendimiento productivo, disminuir la presentación de enfermedades e incrementar el margen de ganancia económica han sido varios de los principales retos de la acuicultura (Oliva-Teles, 2012). Adicionalmente, la creciente industrialización y tecnificación de los procesos productivos en acuicultura, cada vez más intensivos y a gran escala, ha generado un incremento de patologías en diferentes especies de peces (Roberts, 2012). Las enfermedades relacionadas con la nutrición ocupan una parte importante de aquellas que afectan en general a los peces y en este caso las tilapias. Dentro de dichas alteraciones nutricionales están las que generan lesiones degenerativas de diversos órganos (Iregui et al., 2004, Hardy R, 2012). Las observaciones de campo y trabajos preliminares realizados en Colombia han demostrado el incremento de patologías hepáticas en tilapias, donde aquellas relacionadas con alteraciones de tipo hígado graso se han venido convirtiendo en un problema preocupante y poco caracterizado en la producción nacional (Iregui et al., 2004, Rondón-Barragán y Eslava-Mocha, 2009). Múltiples factores se han relacionado con el desarrollo de este tipo de alteraciones, dentro de los cuales pueden mencionarse: 1) deficiencias nutricionales a causa de dietas que no suplen los requerimientos específicos para la especie. 2) Alteraciones del alimento suministrado asociadas a grasas oxidadas y peroxidación lipídica. 3) Uso indiscriminado de fármacos y sustancias tóxicas. 4) Periodos prolongados de hipoxia (Wolf and Wolfe, 2005, Hardy R, 2012, Iregui et al., 2004, Spisni et al., 1998, Boorman et al., 1997). Así, Rey, (2002), en un estudio histopatológico de enfermedades de tilapia, reporta una prevalencia del $78.5 \%$ de vacuolización hepática. Allí se observa un aumento en la severidad 
de estas lesiones con la edad de los peces, pero sin caracterizar si estas vacuolizaciones corresponden a lípidos. Del mismo modo, Rondón-Barragán y Eslava-Mocha, (2009) reportan un $84 \%$ de prevalencia de cambios degenerativos compatibles con hígado graso en tilapias en producciones semi-intensivas, aun cuando no evidenciaban ningún signo de enfermedad en los departamentos del Huila, Meta y Casanare. Dentro de los problemas asociados a nutrición inadecuada se encuentran las alteraciones del metabolismo de los lípidos, debido en gran medida, a una suplementación inadecuada de los perfiles de ácidos grasos requeridos para cada especie de pez (Tocher, 2003, Sargent et al., 1989, Glencross, 2009).

Está bien establecido que los peces requieren varios ácidos grasos poliinsaturados esenciales para el crecimiento y desarrollo normal, incluyendo la reproducción. Entre los más relevantes se encuentran el ácido docosahexaenoico (DHA, 22:6n-3), ácido eicosapentaenoico (EPA, 20:5n-3), ácido araquidonico (ARA, 20:4n-6), ácido linolenico (LNA 18:3n-3) y linoleico (LOA, 18:2n-6) (Glencross, 2009, Tocher, 2003). Los requerimientos de estos lípidos son diferentes entre peces de agua dulce y marinos (Glencross, 2009, Tocher, 2003). Teniendo en cuenta lo anteriormente expuesto se plantea que, aunque es frecuente encontrar cambios degenerativos en los animales que alcanzan un peso óptimo para el mercado, no se sabe con certeza cuál es la relación de los diversos factores que intervienen en la generación de dichas alteraciones y la predisposición al padecimiento de otras enfermedades. Se propuso evaluar la influencia de dos fuentes diferentes de lípidos con diversos porcentajes de inclusión en la dieta sobre el rendimiento productivo y la presentación de hígado graso en tilapias híbridas (Oreochromis spp).

\section{Materiales y métodos}

\section{Peces}

Se utilizaron 396 alevinos de tilapia roja (Oreochromis spp) con un peso de $5.96 \pm 1.09$ gr, obtenidos de un productor local y transferidos al laboratorio de alimentación de peces del Instituto de Acuicultura de la Universidad de los Llanos para aclimatación. Después de este periodo, los peces fueron distribuidos aleatoriamente y en igual número, en 18 tanques circulares de 500 litros con aireadores individuales en un sistema de recirculación cerrado. Permanecieron 15 días en el nuevo sistema antes del período experimental de alimentación. La temperatura del agua fue $27 \pm 1,5^{\circ} \mathrm{C}$ y el oxígeno disuelto osciló entre 5.5 y 6 mg/L.

\section{Dietas experimentales}

Se suministraron 6 dietas semipurificadas isoprotéicas (32\% PB) con 3 porcentajes de lípidos (5, 9 y 13\%), utilizando aceite de pescado o aceite de soya (Sigma-Aldrich,St. Louis,MO,USA) (Tabla 1). Todos los ingredientes fueron mezclados, humedecidos y finalmente procesados en micro-extrusora (Extrutec ${ }^{\circledR}$, Riberão Preto-Brasil), mantenidos en refrigeración durante todo el periodo experimental. Se hizo análisis bromatológico de cada una de las dietas experimentales aplicando la metodología descrita por la Asociación Oficial de Análisis Químicos AOAC, (1995). El perfil de ácidos grasos de cada una de las dietas fue realizado en la Universidad Nacional de Colombia-Laboratorio de Toxicología Animal y se determinó por cromatografía de gases con ionización de llama (Shimadzu GC-20aㅡ). Cada una de las 6 dietas experimentales fue suminis-

Tabla 1. Composición proximal de las dietas experimentales.

\begin{tabular}{lrrrrrr}
\hline \multicolumn{1}{c}{ DIETA } & AP 5 & AP 9 & AP 13 & AS 5 & AS 9 & AS 13 \\
\cline { 1 - 6 } Ingredientes (\% MS) & & & & & & \\
\cline { 1 - 7 } Caseína (a) & 34,8 & 34,8 & 34,8 & 34,8 & 34,8 & 34,8 \\
Gelatina & 3,3 & 3,3 & 3,3 & 3,3 & 3,3 & 3,3 \\
Dextrina (b) & 40,0 & 40,0 & 40,0 & 40,0 & 40,0 & 40,0 \\
Alfa celulosa & 12,5 & 8,5 & - & 12,5 & 8,5 & - \\
Aceite de pescado & 5,0 & 9,0 & 17,5 & - & - & - \\
Aceite de soya & - & - & - & 5,0 & 9,0 & 17,5 \\
Mezcla vitaminas & 0,2 & 0,2 & 0,2 & 0,2 & 0,2 & 0,2 \\
Mezcla macromineral(c) & 4,0 & 4,0 & 4,0 & 4,0 & 4,0 & 4,0 \\
Mezcla micromineral(d) & 0,1 & 0,1 & 0,1 & 0,1 & 0,1 & 0,1 \\
Ácido ascórbico & 0,1 & 0,1 & 0,1 & 0,1 & 0,1 & 0,1 \\
\hline Composición proximal & & & & & & \\
\hline Materia seca (\%) & 68,4 & 68,9 & 68,3 & 70 & 72,5 & 79,4 \\
Proteína bruta (\%) & 32,0 & 32,0 & 32,0 & 32,0 & 32,0 & 32,0 \\
Energía Kcal/Kg & 4513 & 4711 & 4847 & 4671 & 4817 & 4835 \\
Cenizas (\%) & 3,4 & 3,2 & 3,1 & 3,1 & 3,2 & 3,2 \\
Lípidos (\%) & 5,0 & 8,4 & 12,2 & 4,9 & 9,0 & 13,1 \\
\hline
\end{tabular}

(a) Composición: M.S 930, PB 843, lípidos 06; cenizas 36.6. AAE: Arg 34.0, His 25.9, Ile 50.0, Leu 84.6, Lys 81.1 Met 26.7, Phe 45.2, Thr 38.1, Trp 12.1, Val 67.1. AANE: Asp 73.3 Glu 200, Ser 56.7, Pro 60.0, Gly 23.3, Ala 36.7, Tyr 46.0, cis03.1 ( $\left.\mathrm{g} \mathrm{kg}^{-1} \mathrm{~PB}\right)$. NRC 1993.

(b) Composición: M.S 910, PB 940,2, lípidos 22,9; cenizas 36.6. AAE: Arg 69.7, His 07.1, Ile 13.8, Leu 27.4, Lys 35.5, Met 07.3, Phe 17.1, Thr 18.1, Trp 00.1, Val 20.9. AANE: Ac. Asp 60.0, Glu 114, Ser 10, Pro 20.5, Gly 63.5, Ala 25.5, Tyr 01.2, Cys 00.3( $\left.\mathrm{g} \mathrm{kg}^{-1} \mathrm{~PB}\right)$. NRC 1993

(c) VitaminaRovimix ${ }^{\circledR}$ Lab. Roche S.A: Vit. A $8 \times 10^{6}$ UI; Vit. $D_{3}$ $1.8 \times 10^{6} \mathrm{UI}$, Vit. E 66,66 g, Vit. B 6,66 g, Vit. B 13.33 g, Vit. $\mathrm{B}_{6} 6.66 \mathrm{~g}$, Acido Pantoténico $33.33 \mathrm{~g}$, Biotina $533.3 \mathrm{mg}$, Acido Fólico 2.66 g, Acido Ascórbico 400.0 g, Acido Nicotínico 100.0 g, Vit. $B_{12} 20.0 \mathrm{mg}$, Vit. $K_{3} 6.66$ g, filercsp. $1.0 \mathrm{~kg}$ )

(d) Mineral mix ${ }^{\circledR}$ Lab. Roche S.A. 0.5 (Composición para 100 g: Mg 1.0, Zn 16.0, Fe 4.0, Cu 1.0, I 0.5, Se 0.05, Co 0.01; macro-minerales: 40,2 (composición para $100 \mathrm{~g}$ mezcla : $\mathrm{Ca}\left(\mathrm{H}_{2} \mathrm{PO}_{4}\right) 13.6 \mathrm{~g}$; Ca Lactato $34.85 \mathrm{~g} ; 2 \mathrm{MgSO}_{4} .7 \mathrm{H}_{2} \mathrm{O}, 13.2 \mathrm{~g}$; $\mathrm{KH}_{2} \mathrm{PO}_{4} 24 \mathrm{~g} ; \mathrm{NaCl} 4.5 \mathrm{~g} ; \mathrm{AlCl}_{3} 0.015 \mathrm{~g}, \mathrm{CMC}_{4} 9.835 \mathrm{~g}$ ). 
trada a tres grupos replicados que contenían 20 peces cada uno. Se suministraron las dietas dos veces al día (08:00h y $18: 00 h)$ durante 60 días, registrando el consumo de alimento diariamente.

\section{Muestreo}

Al Comenzar el ensayo se muestrearon 36 peces para la evaluación de los parámetros iniciales. Al finalizar el período experimental se registró el peso de todos los peces y se tomaron 10 peces por réplica para evaluación de los parámetros finales. Para histopatología del hígado se tomaron cinco animales de cada réplica. Tanto al inicio como al final se determinaron valores de peso, índice hepatosomático y viscerosomático. Se realizó evaluación de patología macroscópica del hígado e histopatológica. Los peces se anestesiaron con una solución de Eugenol: etanol 1:10 a una dosis de $100 \mathrm{mg} / \mathrm{L}$. Se pesaron con una balanza analítica (Ohaus Traveler, Parsippany,USA). Posteriormente, se sacrificaron los peces mediante sección de la medula. Se realizó disección del hígado y de las vísceras. Se evaluó patología macroscópica del hígado y se registró su peso para índices corporales. Para el análisis microscópico, se tomó la mitad del hígado de cada pez muestreado, se fijó en formalina buferada al 10\% y se realizó procesamiento histológico de rutina. Se realizaron cortes de 3 a $5 \mu \mathrm{m}$ de grosor y se tiñeron con hematoxilina y eosina. Adicionalmente, se realizaron tinciones diferenciales para carbohidratos (Ácido Peryodico de Shiff) y lípidos (Rojo Oleoso).Para esta última se realizaron cortes congelados de los hígados fijados en formalina, siguiendo la metodología propuesta por Luna (1968). La evaluación histopatológica se realizó con un microscopio Nikon ECLIPSE 80i ( Nikon Corporation, Japan).

\section{Cálculo de índices y parámetros productivos}

Los índices corporales se calcularon según la fórmula:

$$
\begin{array}{r}
\text { Índice hepatosomático }=100 *(\text { peso del hígado/ } \\
\text { peso corporal })
\end{array}
$$

$$
\begin{gathered}
\text { Índice viscerosomático }=100 * \text { (peso de las vísceras/ } \\
\text { peso corporal) }
\end{gathered}
$$

Para la evaluación del rendimiento productivo se utilizaron los siguientes parámetros:

$$
\begin{aligned}
& \text { Ganancia de peso }(G P)=(\text { Peso final }- \text { Peso inicial }) \\
& \text { peso inicial ( } g \text { ) } \\
& \text { Incremento de peso (\%) = (peso final-peso inicial) }) \\
& \text { (peso inicial) } \times 100
\end{aligned}
$$

$$
\begin{aligned}
& \text { Tasa de conversión - Materia Seca ofrecida (g)/ } \\
& \text { alimenticia (FCR) = Ganancia de Peso (g) } \\
& \text { Tasa de crecimiento } \quad 100^{*}[\operatorname{Ln} \text { (peso final (g)) } \\
& \text { específico }(S G R \%) \quad=-\operatorname{Ln}(\text { peso inicial }(g))] / \\
& \text { tiempo (días) }
\end{aligned}
$$

\section{Evaluación de las alteraciones patológicas}

Macroscópicamente se evaluó la coloración del hígado y su tamaño con respecto a la cavidad celómica, estableciéndose 4 grados de severidad según los siguientes criterios: Grado 1(Hígado normal): Se observa una coloración roja oscura a caoba sin aumento de tamaño dentro de la cavidad celomica. Grado 2 (Leve): Coloración amarilla translucida con aumento leve de tamaño. Grado 3 (Moderado): Coloración pálida, con aumento moderado del tamaño del hígado y múltiples arborizaciones. Grado 4 (Severo): Coloración pálida intensa con aumento severo de tamaño.

Las lesiones hepáticas microscópicas se caracterizaron según el grado de severidad de la lesión, obedeciendo a los siguientes criterios establecidos por Wolf and Wolfe, (2005) y Boorman et al., (1997): Grado 0 (sin infiltración aparente): Se observa una célula hepática con su contorno visible, núcleo central, con algunas vacuolas pequeñas intracitoplasmáticas que no afectan la forma de la célula ni desplazan el núcleo. Grado 1 (multifocal leve): Se observan múltiples focos de hepatocitos con un contorno visible, núcleo ligeramente desplazado a la periferia y vacuolas intracitoplasmáticas evidentes de tamaño homogéneo que no distorsionan la arquitectura celular. Grado 2 (multifocal moderada): Se observan múltiples focos de hepatocitos con contorno visible, núcleo desplazado a la periferia y múltiples vacuolas intracitoplasmaticas de tamaño homogéneo que no distorsionan la arquitectura celular. Grado 3 (multifocal severa): Múltiples focos de hepatocitos que pierden el contorno celular, núcleo severamente desplazado a la periferia o imperceptible y vacuolas intracitoplasmaticas que coalescen hasta formar un citoplasma homogéneo que distorsionan totalmente la arquitectura celular. Grado 4 (generalizada leve): Se observan vacuolizaciones en toda el área examinada del órgano, con hepatocitos de contorno visible, núcleo ligeramente desplazado a la periferia y vacuolas intracitoplasmáticas evidentes de tamaño homogéneo que no distorsionan la arquitectura celular. Grado 5 (Generalizada moderada): Se observan vacuolizaciones en toda el área examinada del órgano, con hepatocitos con contorno visible, núcleo desplazado a la periferia y múltiples vacuolas intracitoplasmáticas de tamaño homogéneo que no distorsionan la 
arquitectura celular. Grado 6 (Generalizado severo): Se observan vacuolizaciones en toda el área examinada del órgano, con hepatocitos que pierden el contorno celular, núcleo evidentemente desplazado a la periferia o imperceptible y vacuolas intracitoplasmaticas que coalescen hasta formar un citoplasma homogéneo que distorsionan totalmente la arquitectura celular.

\section{Análisis estadístico}

Los resultados obtenidos en variables de desempeño productivo e índices corporales se evaluaron aplicando un análisis de varianza ANOVA de una vía. Se utilizó la prueba de Kolmogorov-Smirnov y Levene para evaluar homogeneidad de varianzas, y adicionalmente se utilizó la prueba de Tukey-Kramer para evaluar diferencias significativas entre tratamientos. En estas evaluaciones se utilizó el programa SAS, versión 8.05 y GraphPad Prism 5.03. Las lesiones halladas al examen de necropsia y en histopatología fueron descritas y expresadas en grados de severidad de la lesión, y evaluadas mediante el test de Kruskal-Wallis.

\section{Resultados}

\section{Parámetros productivos $e$ índices corporales}

Se observó una mayor ganancia de peso, crecimiento y eficiencia alimenticia en los animales alimentados con las dietas que contenían aceite de soya respecto a las que contenían aceite de pescado, con diferencias esta- dísticamente significativas $(\mathrm{P}<0,05)$. No se observaron diferencias estadísticamente significativas entre los porcentajes de inclusión de lípidos para cada fuente utilizada. Los resultados de parámetros productivos se muestran en la Tabla 2 y Figura 1. El índice hepatosomático de los peces alimentados con la dieta que contenía aceite de soya en un porcentaje de inclusión del 13\% mostró disminución significativa $(p<0,05)$ con respecto a los peces alimentados con las dietas que contenían 5 , 9 y $13 \%$ de aceite de pescado (Tabla3). El índice viscerosomático no arrojó diferencias significativas $(p>0,05)$ en ninguno de los porcentajes de inclusión evaluados de los aceites en todos los peces (Tabla 3).

\section{Perfil de ácidos grasos de las fuentes de lípidos evaluadas}

La composición de ácidos grasos de las fuentes de lípidos evaluadas se muestra en la Tabla 5. En general, las dietas que contenían aceite de pescado presentaron un mayor porcentaje de ácidos grasos n-3 (23,12\%) comparado con las dietas que contenían aceite de soya $(7,05 \%)$. Por el contrario, las dietas que contenían aceite de soya presentaban un mayor porcentaje de ácidos grasos n-6 (53,47 \%) comparado con las dietas que contenían aceite de pescado (5,07\%).

\section{Patología macroscópica y microscópica}

Macroscópicamente, las características morfológicas observadas fueron principalmente cambios de coloración en el hígado, variando desde un amarillo pálido,

Tabla 2. Parámetros productivos evaluados en tilapias alimentadas durante 60 días con dietas que contenían aceite de soya y de pescado como fuente de lípidos en diferentes tasas o niveles de inclusión.

\begin{tabular}{|c|c|c|c|c|c|c|c|}
\hline \multicolumn{8}{|c|}{ Parametros productivos } \\
\hline Valores medios & INICIO & $5 \%$ AP & 9\%AP & $13 \%$ AP & $5 \%$ AS & $9 \%$ AS & $13 \%$ AS \\
\hline Peso individual inicial & $5,96 \pm 1,09^{a}$ & & & & & & \\
\hline Peso individual final & & $30,61 \pm 9,93^{b, c}$ & $21,74 \pm 4,82^{b}$ & $25,6 \pm 7,10^{b}$ & $39,91 \pm 12,76^{c, d}$ & $37,22 \pm 10,47, \mathrm{~d}$ & $38,07 \pm 12,73^{d}$ \\
\hline Ganancia peso (gr/pez) & & $23,5 \pm 7,0^{a}$ & $17,6 \pm 3,5^{b}$ & $19,5 \pm 1,4^{a, b}$ & $33,8 \pm 8,2^{c}$ & $30,9 \pm 3,7^{\mathrm{c}}$ & $32,1 \pm 7,4^{\mathrm{c}}$ \\
\hline Incremento peso \% & & $393,8 \pm 117,7^{a}$ & $262 \pm 21,7^{b}$ & $327 \pm 24,1^{a, b}$ & $566 \pm 137,9^{c}$ & $519 \pm 62,2^{c}$ & $538 \pm 124^{c}$ \\
\hline $\begin{array}{l}\text { Tasa especifica de } \\
\text { crecimiento (SGR) }\end{array}$ & & $3,17 \pm 0,66^{\mathrm{a}, \mathrm{d}}$ & $2,54 \pm 0,45^{b}$ & $2,84 \pm 0,58^{a, b}$ & $3,67 \pm 0,74^{c}$ & $3,57 \pm 0,65^{a, c, d}$ & $3,58 \pm 0,74^{\mathrm{a}, \mathrm{c}, \mathrm{d}}$ \\
\hline $\begin{array}{l}\text { Tasa de conversión } \\
\text { alimenticia (FCR) }\end{array}$ & & $0,98 \pm 0,42^{a, b, c}$ & $1,21 \pm 0,38^{a, b}$ & $1,25 \pm 0,67^{a, b}$ & $1,15 \pm 0,79^{b, c}$ & $1,01 \pm 0,66^{c}$ & $0,95 \pm 0,53^{c}$ \\
\hline
\end{tabular}

Letras diferentes indican diferencias significativas $(p<0,05)$

Tabla 3. Índices corporales evaluados en tilapias alimentadas durante 60 días con dietas que contenían aceite de soya y de pescado como fuente de lípidos en diferentes tasas o niveles de inclusión. IHS: Índice Hepatosomático, IVS: Índice viscerosomático.

\begin{tabular}{cccccccc}
\hline \multicolumn{7}{c}{ Indices corporales } \\
\hline Índice & INICIO & $\mathbf{5 \% A P}$ & $\mathbf{9 \% A P}$ & $\mathbf{1 3 \% A P}$ & $\mathbf{5 \% A S}$ & $\mathbf{9 \% A S}$ & $\mathbf{1 3 \% A S}$ \\
\hline IHS & $2,533 \pm 0,729^{\mathrm{a}, \mathrm{b}, \mathrm{c}}$ & $3,216 \pm 1,425^{\mathrm{a}, \mathrm{b}}$ & $3,291 \pm 1,199^{\mathrm{a}, \mathrm{b}}$ & $3,122 \pm 0,9705^{\mathrm{a}, \mathrm{b}}$ & $2,491^{\mathrm{c}, \mathrm{b}} \pm 0,6804$ & $2,456 \pm 0,9293^{\mathrm{c}, \mathrm{b}}$ & $1,99 \pm 0,8997^{\mathrm{c}}$ \\
IVS & $8,504 \pm 1,09^{\mathrm{a}, \mathrm{b}}$ & $8,186 \pm 1,91^{\mathrm{a}}$ & $8,386 \pm 2,34^{\mathrm{a}, \mathrm{b}}$ & $8,939 \pm 2,42^{\mathrm{a}, \mathrm{b}}$ & $9,973 \pm 2,31^{\mathrm{a}, \mathrm{b}}$ & $9,613 \pm 1,58^{\mathrm{a}, \mathrm{b}}$ & $9,488 \pm 2,09^{\mathrm{a}, \mathrm{b}}$ \\
\hline
\end{tabular}

Letras diferentes indican diferencias significativas $(p<0,05)$ 


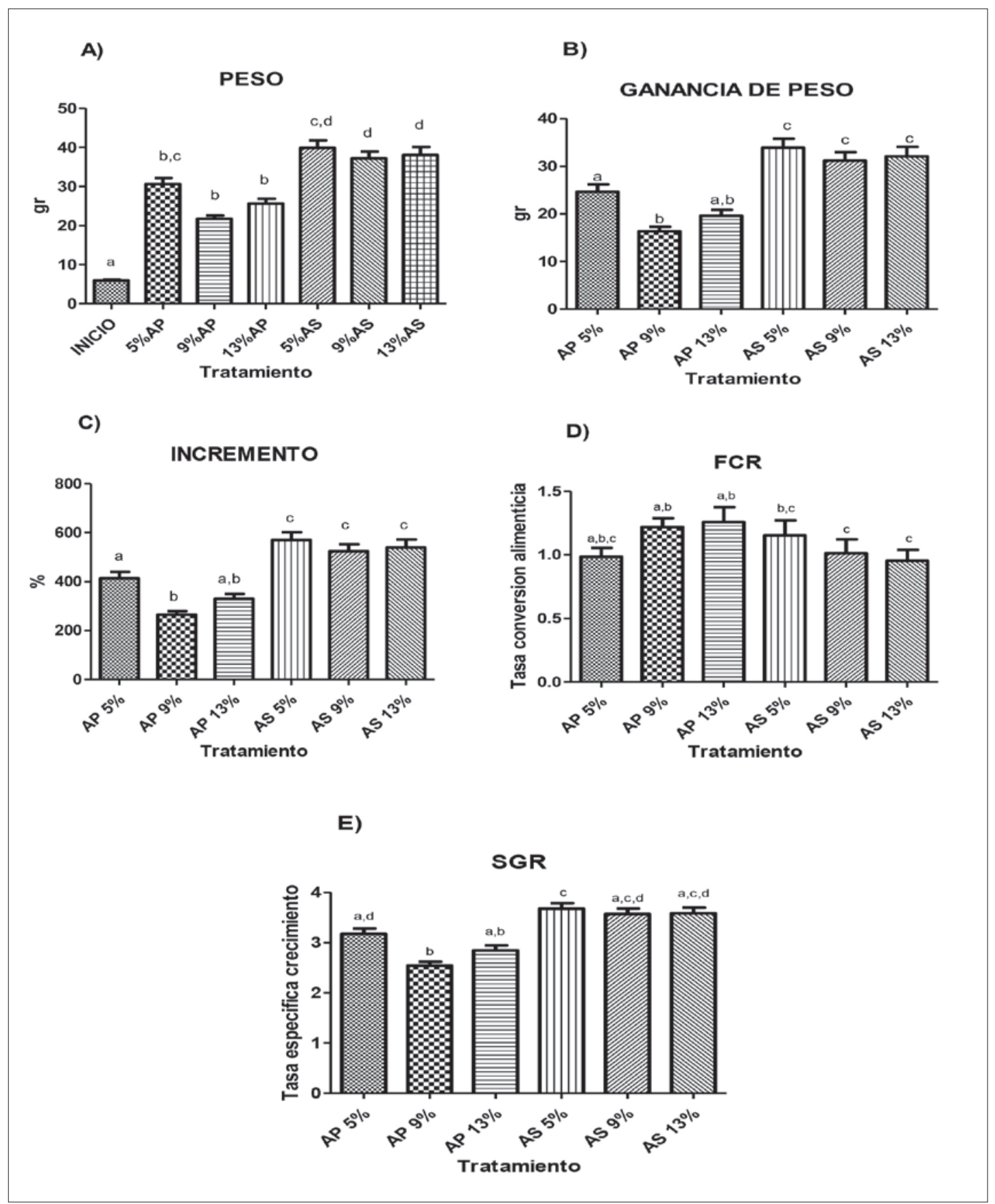

Figura 1. Parámetros de desempeño productivo en tilapias alimentadas con dietas que contenían aceite de soya y de pescado como fuentes de lípidos en diferentes porcentajes durante 60 días. A) Peso final e inicial, B) Ganancia de peso, C) Incremento, D) Tasa de conversión alimenticia (FCR), E) Tasa de crecimiento específica $(S C R)$. Letras diferentes indican diferencias significativas $(p<0,05)$.

con arborizaciones que abarcaban todo el parénquima hepático, hasta un color blanquecino intenso, con aumento del tamaño del hígado en peces alimentados, ya sea con aceite de pescado o con aceite de soya (Figura 3). Se observó un grado de lesión macroscópica de menor severidad $(\mathrm{p}<0.05)$ en los peces tratados con 9 y $13 \%$ de aceite de soya, presentando un grado 1 y 2 de lesión, en promedio, mientras que los trata- mientos del 5 y $9 \%$ de aceite de pescado presentaban grados de lesión entre 3 y 4 . (Tabla 4 y Figura 2). Microscópicamente, la degeneración vacuolar hepática fue significativamente menor $(p<0,05)$ en los peces alimentados con las dietas que contenían 5,9 y $13 \%$ de aceite de soya con respecto a los peces alimentados con dietas que contenían 9 y $13 \%$ de aceite de pescado. Los animales que fueron alimentados con 


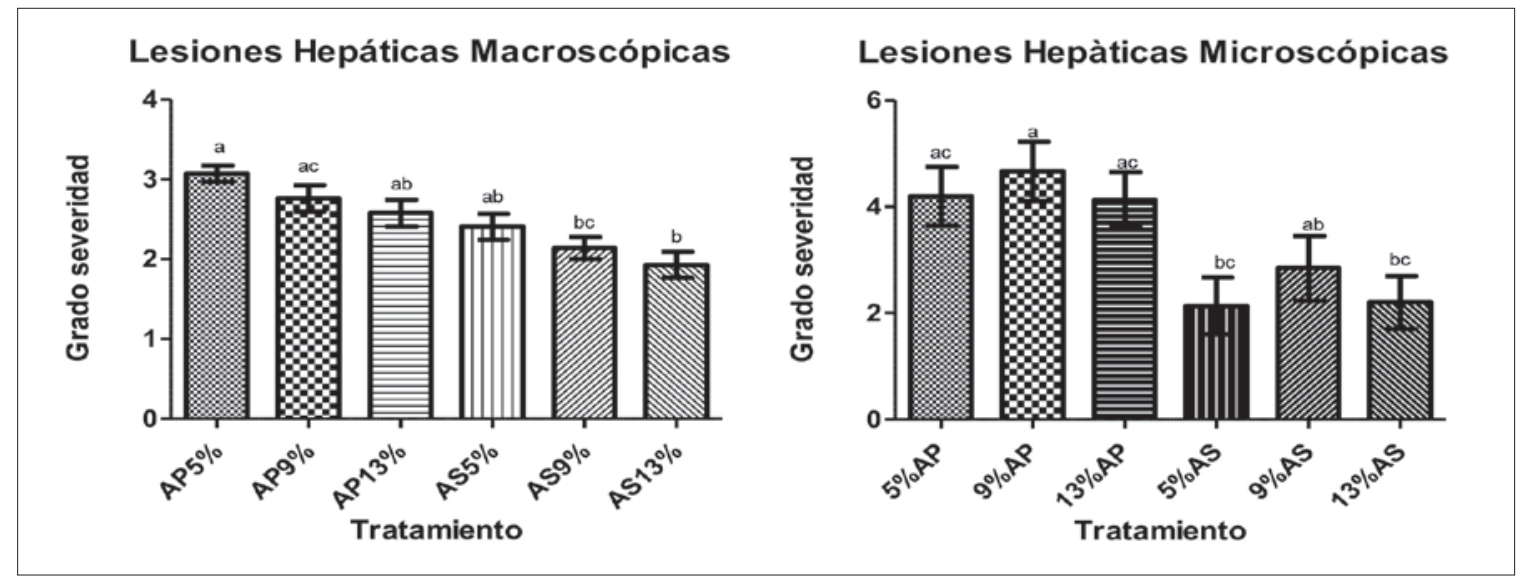

Figura 2. Lesiones hepáticas macroscópicas y microscópicas de tilapias alimentadas durante 60 días con dietas que contenían aceite de soya y de pescado en diferentes porcentajes de inclusión como fuente de lípidos. Letras diferentes indican diferencias significativas $(p<0,05)$.

Tabla 4. Promedio del grado de lesión macroscópica y microscópica en el hígado de tilapias alimentadas durante 60 días con dietas que contenían aceite de soya y de pescado con diferentes niveles de inclusión.

\begin{tabular}{lcccccc}
\cline { 2 - 6 } & \multicolumn{5}{c}{ Lesiones Hepáticas } \\
\cline { 2 - 6 } & $\mathbf{5 \% A P}$ & $\mathbf{9 \% A P}$ & $\mathbf{1 3 \% A P}$ & $\mathbf{5 \% A S}$ & $\mathbf{9 \% A S}$ & $\mathbf{1 3} \%$ AS \\
\hline Lesiones macroscópicas & $3,07 \pm 0,53^{\mathrm{a}}$ & $2,76 \pm 0,76^{\mathrm{a}, \mathrm{c}}$ & $2,57 \pm 0,85^{\mathrm{a}, \mathrm{b}}$ & $2,40 \pm 0,84^{\mathrm{a}, \mathrm{b}}$ & $2,13 \pm 0,74^{\mathrm{b}, \mathrm{c}}$ & $1,92 \pm 0,85^{\mathrm{b}}$ \\
Lesiones Microscópicas & $4.2 \pm 2,11^{\mathrm{a}, \mathrm{c}}$ & $4.7 \pm 1,92^{\mathrm{a}}$ & $4.1 \pm 1,99^{\mathrm{a}, \mathrm{c}}$ & $2.1 \pm 2,06^{\mathrm{b}, \mathrm{c}}$ & $2.8 \pm 2,19^{\mathrm{a}, \mathrm{b}}$ & $2.2 \pm 1,93^{\mathrm{b}, \mathrm{c}}$ \\
\hline
\end{tabular}

Letras diferentes indican diferencias significativas por la prueba de Kruskal-Wallis $(\mathrm{P}<0.05)$

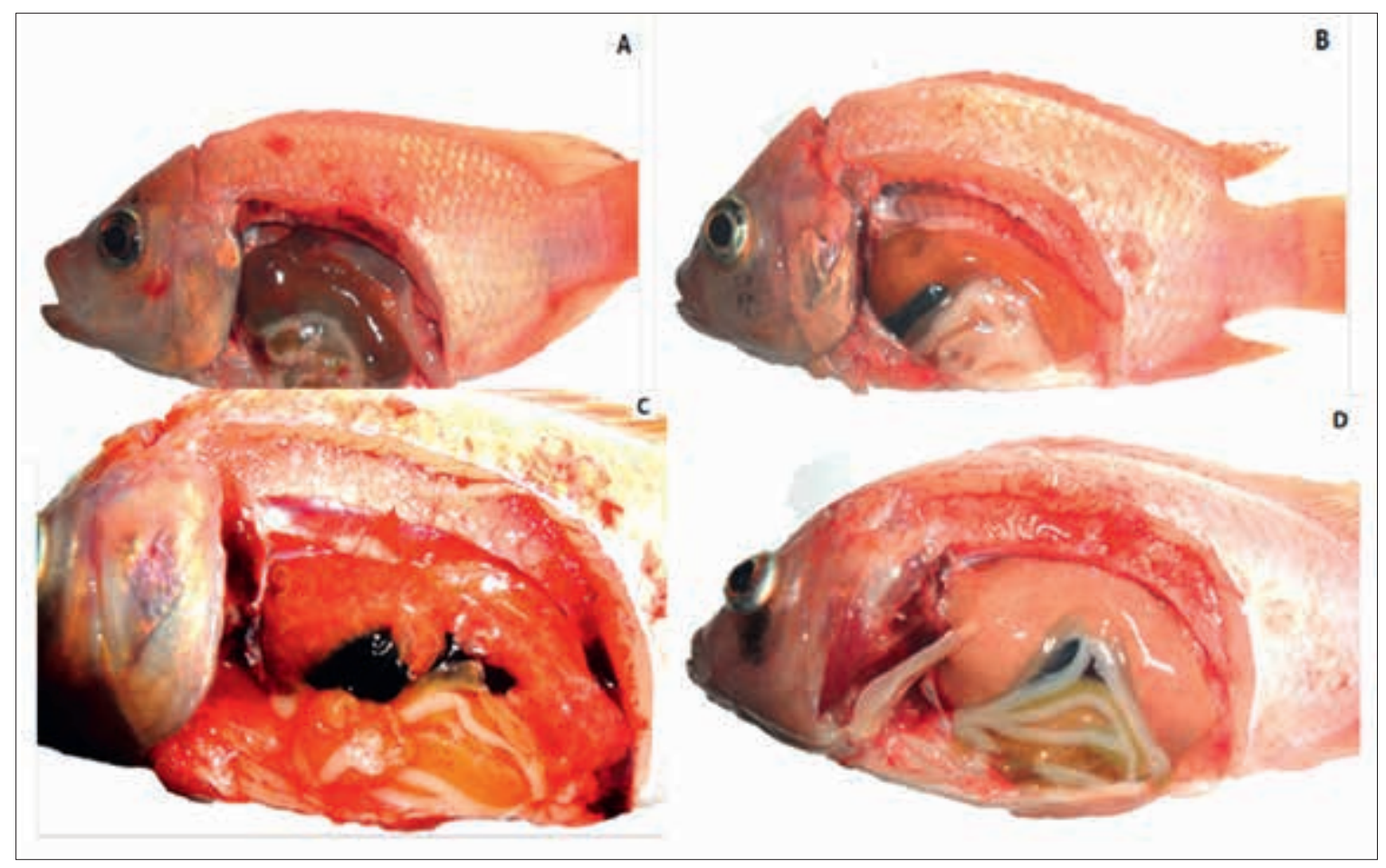

Figura 3. Patrones morfológicos de lesión macroscópica en el hígado de tilapias alimentadas con dietas con diferentes fuentes de lípidos. A) Hígado de coloración y tamaño normal (Grado 1); B) Hígado con cambio de coloración hacia un amarillo pálido con leve aumento de tamaño. (Grado 2) C) Hígado moderadamente aumentado de tamaño y evidente patrón arborescente generalizado en el parénquima hepático (Grado 3). D) Aumento severo del tamaño del hígado (hepatomegalia) y cambio de coloración difusa hacia un tono blanquecino (Grado 4). 


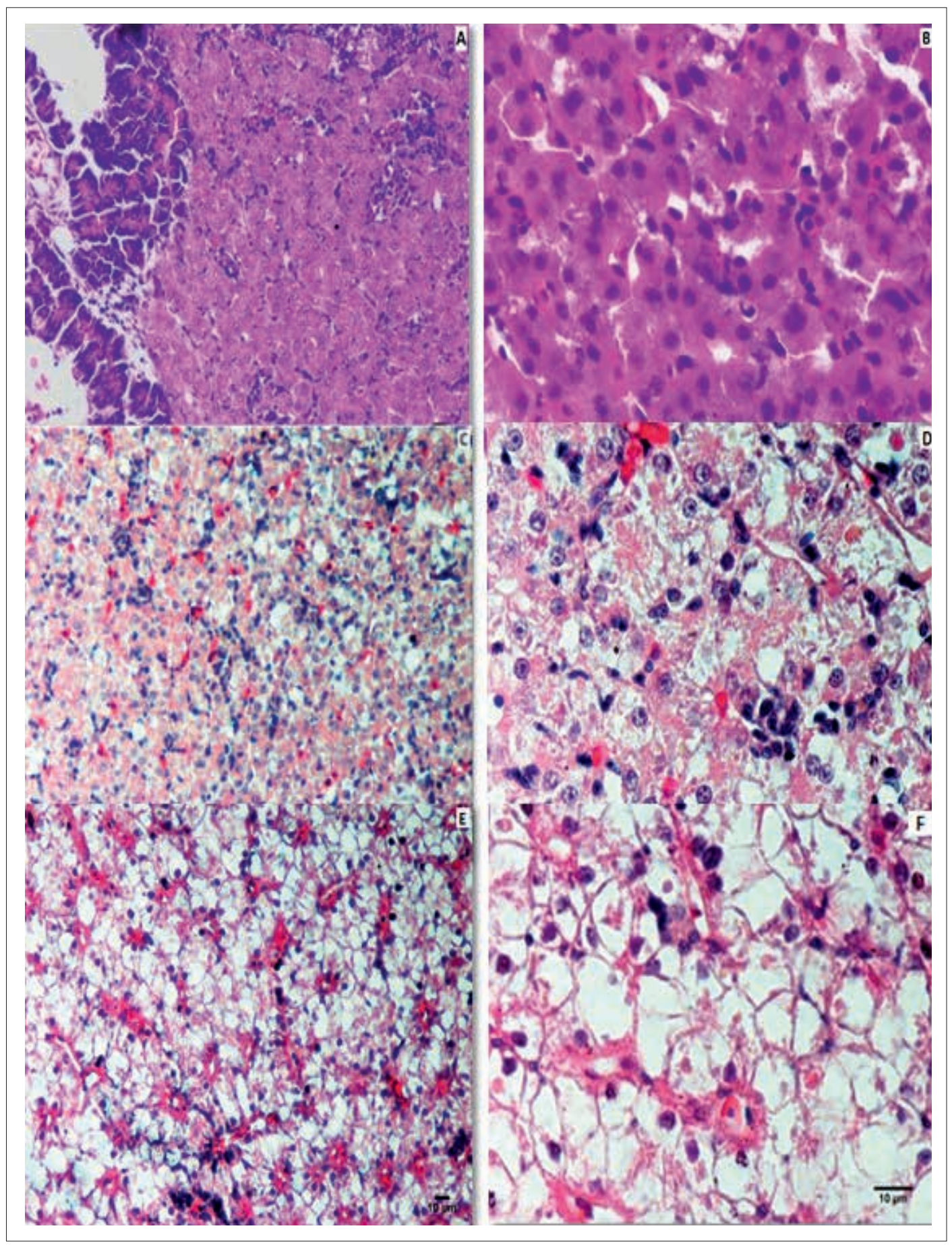

Figura 4. Patrones morfológicos de lesión (degeneración vacuolar) a nivel microscópico, en el hígado de tilapias alimentadas con dietas con diferentes fuentes y porcentajes de inclusión de lípidos. A) Hígado de tilapia aparentemente normal, nótese las células con un citoplasma homogéneo, eosinófilo y núcleo central (asterisco) (40X). B) acercamiento de los hepatocitos normales del hígado de tilapia (100X). C) Degeneración vacuolar leve a moderada. Se observan focos de células vacuoladas con citoplasma claro con leve desplazamiento del núcleo seguido de una zona de transición de hepatocitos aparentemente normales (40X). D) acercamiento en donde se observa claramente la vacuolización del citoplasma de los hepatocitos sin deformar el contorno celular y desplazamiento leve del núcleo y algunos hepatocitos con citoplasma granular homogéneo aparentemente normal (100X). E) Degeneración vacuolar severa. Se observa una severa vacuolización del citoplasma, desplazamiento del núcleo hacia la periferia y distorsión del contorno de los hepatocitos (40X). F) En algunos casos las vacuolas coalescen unas con otras y forman grandes agregados de restos del citoplasma celular de varias células (100x). (Tinción con HyE) 
aceite de pescado, en todos los niveles de inclusión, presentaron un grado de severidad de lesión de 4.3 en promedio, correspondiendo a vacuolizaciones en toda el área examinada del órgano, con hepatocitos de contorno visible, núcleo ligeramente desplazado a la periferia y vacuolas intracitoplasmáticas evidentes de tamaño homogéneo que no distorsionan la arquitectura celular. Los animales que fueron alimentados con aceite de soya, en todos los niveles de inclusión, presentaron un grado de severidad de lesión de 2.3 en promedio, correspondiendo a múltiples focos de hepatocitos con contorno visible, núcleo desplazado a la periferia y múltiples vacuolas intracitoplasmaticas de tamaño homogéneo que no distorsionan la arquitectura celular (Tabla 4 y Figura 2). Con las tinciones diferenciales se encontró vacuolizaciones que contenían material correspondiente a carbohidratos (posiblemente glucógeno) y lípidos. Para los carbohidratos se evidenció en los grados menores de severidad para los dos tratamientos, una distribución granular muy fina que se organizaba en medialunas en la periferia de los hepatocitos, si se compara con los grados de severidad más altos donde la deformación de la célula era evidente, con formación de aglomerados de carbohidratos a manera de cristales refringentes que, en ocasiones, coalescian con cristales adyacentes ( $\mathrm{Fi}$ gura 5 A y B). En la tinción de Red Oil para lípidos se evidenciaron múltiples gotas lipídicas de diversos tamaños, dentro y fuera de los hepatocitos, variando en número y tamaño de acuerdo a la severidad de las lesiones. Las gotas lipídicas fueron menos y más pequeñas en las lesiones de grado 1 a 2 . En los grados de severidad 5 y 6 se evidenciaron mayor cantidad de gotas lipídicas de gran tamaño que en muchos casos coalescian y formaban una gran vacuola que alcanzaba a copar toda la célula (Figura 5 C y D).

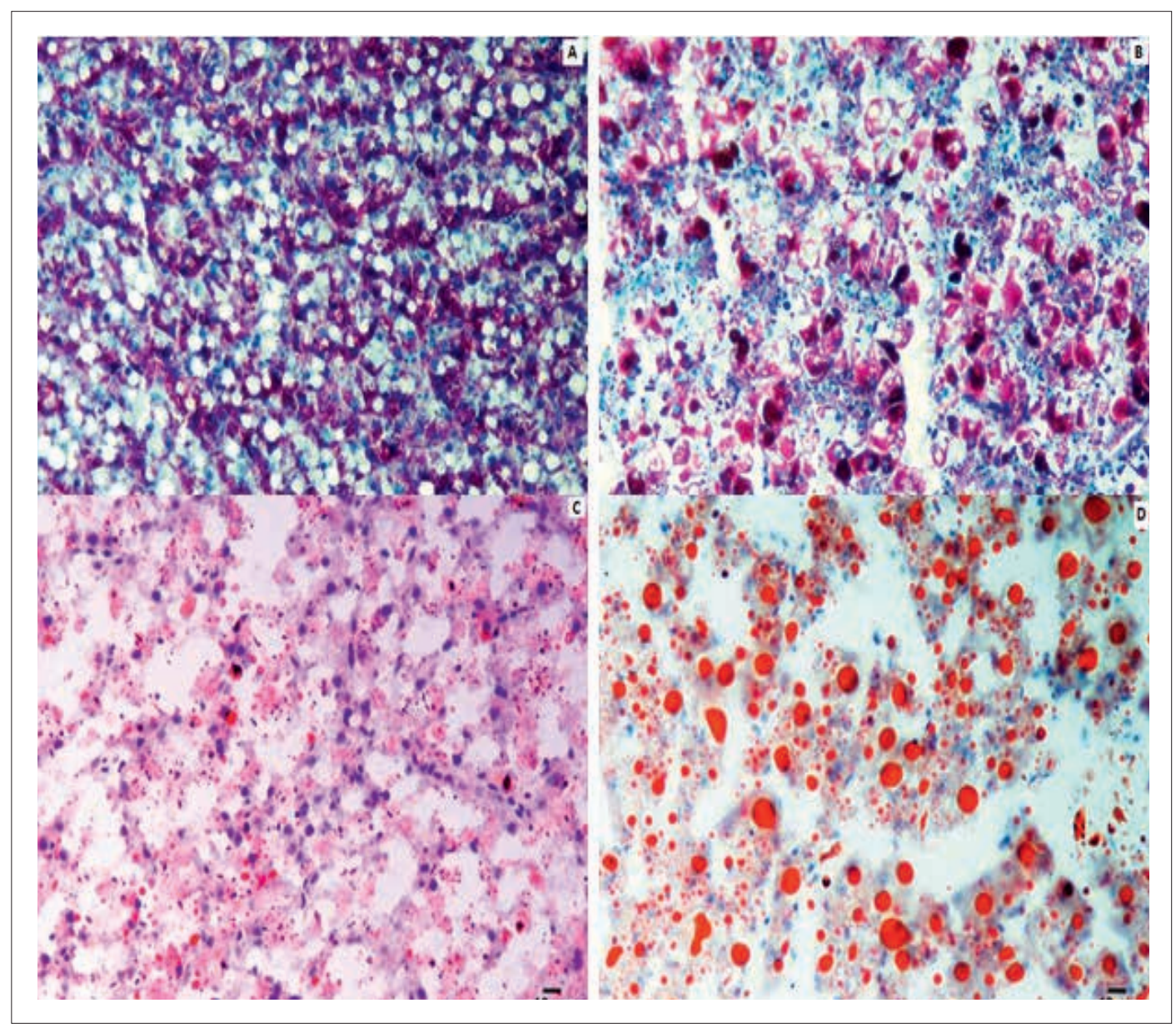

Figura 5. Tinción diferencial para carbohidratos (Ácido peryodico de shiff PAS) y lípidos (Red Oil O) donde se demuestra la presencia tanto de carbohidratos como de lípidos dentro de los hepatocitos. A) Distribución normal del glucógeno dentro de los hepatocitos con múltiples vacuolas lipídicas (Tinción de PAS 40X). B) Vacuolización severa de los hepatocitos con formación de aglomerados de glucógeno intracelulares y deformación severa del contorno celular (Tinción de PAS 40X). C) Múltiples gotas lipídicas de pequeño tamaño dentro de los hepatocitos (tinción con Red Oil O 40X). D) Múltiples gotas lipídicas de gran tamaño que coalescen en varios casos y deforman el contorno celular (tinción con Red Oil O 40X) 


\section{Discusión}

\section{Parámetros productivos}

Según el análisis por cromatografía de gases realizado en este caso, el contenido de ácido linoléico y linolénico en la dieta que contenía aceite de pescado era mucho menor comparado con la dieta que contenía aceite de soya y la proporción n-6/n-3 fue mayor en las dietas que contenían aceite de soya. Los autores no conocen el requerimiento de ácido linolénico (C18:3n-3) para tilapias. Sin embargo, se ha determinado que este es necesario para un óptimo crecimiento junto con el linoléico (C18:2n-6) (Lim et al., 2011, Al-Souti et al., 2012). El menor porcentaje de ácido linoleico en las dietas con aceite de pescado puede explicar el menor rendimiento productivo observado, comparado con las dietas que contenían aceite de soya Sin embargo, estos porcentajes están dentro de los requerimientos necesarios para la especie. Lo anterior indica que hay otros factores involucrados.

Por otro lado, el porcentaje de inclusión de lípidos parece no influir en el rendimiento productivo como se demuestra en este trabajo, teniendo en cuenta que están dentro del rango aceptable y se suplen los requerimientos mínimos de ácidos grasos para la especie como ha sido establecido por (Kanazawa et al., 1980). Los ácidos linoléico (18:2 n-6) y linolénico (18:2 n-3) son esenciales para el crecimiento de los peces de agua dulce, teniendo un mayor efecto el ácido linoléico, como lo han reportado (Kanazawa et al., 1980, Takeuchi et al., 1983, Lim et al., 2011, Olsen et al., 1990). Por otro lado, (Kanazawa et al., 1980, Takeuchi et al., 1983, Aguiar et al., 2007) han reportado que un nivel excesivo de 18:3n-3 o 5\% de aceite de hígado de pescado (pollock Pollachius virens) alto en 20:5n-3 o 22:6 n-3 en dietas que son muy deficientes en n- 6 pueden llevar a disminución del crecimiento. Aguiar et al., (2007) encontraron que se puede incrementar marcadamente la cantidad de EPA y DHA en el hígado de la tilapia nilótica alimentada con aceite de canola como fuente de lípidos, incrementando la cantidad de ácido linolenico (18:3n-3). Ello indica una síntesis endógena a partir de precursores como ácido linoleico y linolenico. Por otra parte, Huang et al., (1998) reportan un mayor crecimiento en tilapias híbridas alimentadas con aceite de pescado, si se compara con el de soya utilizando un porcentaje de inclusión del $8 \%$. Esto indica que no solo el ácido linoléico (18:2 n-6) sino también el linolénico (18:2 n-3), ácido docosahexaenoico (DHA) y ácido eicosapentaenoico (EPA) son requeridos para un óptimo crecimiento (Tocher, 2003, Huang et al., 1998, Olsen et al., 1990), en contraste con los resultados obtenidos en este trabajo.
Además de lo anterior, Al-Souti et al.,(2012) reportan que la ganancia de peso disminuía en tilapias híbridas alimentadas con aceite de maíz (rico en ácido linoleico) y de pescado, a medida que se aumentaba el porcentaje de inclusión de aceite de pescado, mostrando así hallazgos similares a los encontrados en este trabajo. (Olsen et al., 1990, Huang et al., 1998) reportan que las tilapias son capaces de utilizar ácido linoléico como substrato para elongación de ácidos grasos poliinsaturados de cadena larga, más que el linolenico. El-Husseiny et al., (2010) encontraron que la FCR y el rendimiento mejoró significativamente cuando se alimentaron tilapias del Nilo con aceite de soya como fuente de lípidos. Adicionalmente, observaron que se aumenta el porcentaje de supervivencia cuando se incrementa la concentración de ácido linoleico 18:2 n-6 en la dieta, debido a la conversión de este ácido graso a acido araquidónico, que cumple un papel importante en la resistencia al estrés y en la respuesta inmune. Las tilapias, como otros peces de aguas cálidas, están más inclinadas a requerir mayores cantidades de ácidos grasos n-6, comparado con n-3 para un mayor crecimiento, respaldado por el hecho de que altos niveles de n-3 PUFA disminuyen su crecimiento. Hay evidencias de mayor actividad de desaturación cuando las tilapias son alimentadas con aceites de origen vegetal y de la conversión de ácidos grasos de cadena corta y saturados a ácidos grasos de cadena larga insaturados (El-Husseiny et al., 2010, Teoh et al., 2011, Huang et al., 1998). Adicionalmente, es evidente una conversión endógena para satisfacer requerimientos basales, basada en el hecho de que se observan mayores concentraciones de araquidónico en la carcasa cuando se aumentan los niveles de linoléico en la dieta, mientras que los niveles de EPA, DHA y linolénico se incrementan cuando se aumentan los niveles de linolénico en la dieta (Teoh et al., 2011). A pesar de un exceso de linoleico, altos niveles de linolenico, pueden disminuir la conversión de linoleico a araquidónico debido, posiblemente, a la competencia entre linolenico y linoleico, ya que los dos son substratos para la misma enzima D6 desaturasa (El-Husseiny et al., 2010). El-Husseiny et al., (2010) concluyen que el uso de una tasa n6/n3 apropiada en la dieta es más importante para las tilapias que el uso de niveles absolutos de estos ácidos grasos. Así se indica que una tasa LA/LNA menor de 13 es recomendada para alevinos de tilapia. Esto soporta lo encontrado en este trabajo, en donde no se observaron diferencias estadísticas significativas en los parámetros productivos para los porcentajes de inclusión en el aceite de soya. Su tasa n6/n3 estaba dentro del límite del 13\% y con los porcentajes de inclusión evaluados se cumplían los requerimientos necesarios para la especie. 
Adicionalmente esto, explica por qué el aceite de soya es mejor que otros aceites vegetales para la nutrición de estos peces. Lim et al., (2011) proponen que los carbohidratos digestibles pueden ser usados para reemplazar los lípidos dietarios como fuente de energía si los requerimientos de ácidos grasos esenciales son suplidos en tilapias, disminuyendo el uso de aceites de origen animal.

En los peces alimentados con aceite de pescado (Zebrafish y tilapia nilótica) se disminuye la actividad $\Delta 6$ desaturasa, mientras que en los animales alimentados con dietas que contenían aceite vegetal rico en 18:3n3 se incrementa la actividad desaturasa (El-Husseiny et al., 2010). En este incremento, el factor primario está dado por la reducción en la inhibición por producto, dada en gran medida por la considerable reducción de n-3 HUFAS en las dietas que contenían aceite vegetal, más que la provisión de mayor cantidad de PUFAS como sustrato por primera vez (Tocher et al., 2001a). La tasa de conversión de C:18 PUFAS a PUFAS de cadena larga (C:20 y C:22) parece estar influenciada por el nivel de estos preformados en la dieta y en los tejidos del pez.

La presencia de altas concentraciones de C:20 y C:22 preformados y las tasas de conversión de C:18 PUFA a derivados de cadena larga es menor. Lo anterior indica que las enzimas para la conversión están presentes, pero son inhibidas por las altas concentraciones de PUFAS de cadena larga. De este modo, esto puede disminuir el efecto ahorrador de proteína que los lípidos presentan, ya que no son usados como substrato selectivo para la B-oxidación, utilizándose proteína y tejido muscular para producir energía. Por lo tanto, se disminuye el rendimiento productivo (Tocher et al., 2001a, Al-Souti et al., 2012). Adicionalmente, Teoh et al., (2011) encontraron que las tilapias rojas (hibridas) favorecen la B-oxidación de 18:2n-6 y 18:3n3 cuando son alimentadas con aceites vegetales. Por esto disminuyen la acumulación de lípidos en hígado y optimizan el rendimiento productivo ejerciendo un efecto ahorrador de proteína. Por lo anterior, puede explicarse la menor ganancia de peso de los animales que fueron sometidos a la dieta con aceite de pescado, las cuales presentaron un alto contenido de PUFAS de cadena larga. Por otro lado, Asdari et al., (2011) encontraron que Pangasius hypophthalmus presentan un mejor rendimiento productivo cuando son alimentados con aceite de soya y aceite de palma crudo, comparado con aceite de pescado y de linaza. En este estudio no se observaron diferencias significativas en el IHS, entre las diferentes fuentes de lípidos utilizadas.

\section{Lesiones hepáticas}

Las alteraciones hepáticas observadas pueden explicarse con base a los diferentes perfiles de ácidos grasos que tienen cada una de las fuentes de lípidos utilizadas (Tabla 5). Para los tratamientos con dietas que contenían aceite de pescado se encontró mayor proporción de ácidos grasos n-3 que de n-6, siendo estos en gran medida ácidos grasos poliinsaturados de cadena larga.

Se ha encontrado que altas concentraciones de PUFAS de cadena larga inhiben las elongasas y desaturasas en los peces de agua dulce y también ensalmones, limitando la formación de PUFAS endógenos y su incorporación a las membranas celulares Eso genera como consecuencia la acumulación de los PUFAS provenientes de la dieta (Tocher et al., 2001a, Sargent et al., 1989, Al-Souti et al., 2012).

El ácido docosahexaenoico (DHA) 22:6 n-3 actúa de menor manera como substrato para la $\beta$-oxidación mitocondrial, comparado con el ácido linoléico 18:2 n-6 y el ácido oléico 18:1 n-9 en células musculares y hepáticas de tilapias (Szabo et al., 2011). Esto promueve la acumulación de PUFAS de cadena larga en hígado, y de triglicéridos y fosfolípidos en los tejidos en general (Sargent et al., 1989, Bandarra et al., 2011). Aunado a lo anterior, se ha reportado una disminución de la síntesis de VLDL por el hígado en dietas altas en PUFAS de cadena larga, DHA y EPA, en salmones y truchas (Tocher et al., 2001b, Szabo et al., 2011). De esta manera se impide la exportación de lípidos desde el hígado a la sangre, y otros tejidos para su utilización.

También se ha propuesto que el efecto oxidativo ejercido por altas concentraciones de PUFAS n-3 en el hígado, puede conducir a una lipidosis causada principalmente por peroxidación lipídica, oxidación de las lipoproteínas y alteración de la exportación de triglicéridos y otros lípidos desde las células hepáticas a la sangre y otros tejidos (Szabo et al., 2011, Gang et al., 2006). Aunque las dietas utilizadas por nosotros permanecieron refrigeradas durante todo el periodo experimental, disminuyendo la probabilidad de formación de peróxidos lípidicos, no fue posible descartar su presencia en las dietas suministradas como factor influyente en la presentación de lípidosis hepática.

Se ha propuesto que hay una gran síntesis y deposición de triglicéridos en vacuolas cuando los lípidos de la dieta o la cantidad de energía exceden la capacidad de las células hepáticas para oxidar los ácidos grasos, o cuando la síntesis de proteína está alterada o impedida. Así se conlleva al hígado graso (Caballero et al., 2004). En este trabajo, las dietas suministradas cumplían los requerimientos de proteína y energía para la especie. 
Los porcentajes de lípidos no estaban por fuera de los rangos requeridos, siendo poco probable un desequilibrio de energía el que condujera a la lipidosis, más que una alteración en los requerimientos de ácidos grasos esenciales y sus concentraciones, en las diferentes fuentes de lípidos. Las alteraciones encontradas en la distribución del glucógeno en los hepatocitos han sido reportadas en peces sometidos a periodos prolongados de estrés y a diversas hepatotoxinas (Wolf and Wolfe, 2005). En este trabajo se observó una aglomeración de glucógeno principalmente en aquellos tratamientos en donde se observaron mayores grados de degeneración y vacuolización de hepatocitos. Sin embargo, no hay evidencias que soporten un aumento de la cantidad de glucógeno dentro de las células hepáticas. Tampoco si, por el contrario, estos conglomerados de carbohidratos son formados a partir de la ruptura y deformación de las células hepáticas con la posterior agregación y formación del glucógeno de varias células, al ser normal la cantidad de glucógeno intracelular.

Está bien definido que el aceite de pescado como fuente de lípidos es fundamental para peces marinos y que su remplazo total por fuentes de origen vegetal conlleva a alteraciones metabólicas y lesiones hepáticas (Caballero et al., 2004, Caballero et al., 1999, Spisni et al., 1998, Gang et al., 2006). Por otro lado, los estudios que describen la relación entre las fuentes de lípidos, los perfiles de ácidos grasos y la relación con la presentación de alteraciones hepáticas son escasos en peces de agua dulce (Sales y Glencross, 2011) y nulos en tilapias. Como se demuestra en este trabajo, la presentación y la severidad de alteraciones hepáticas de tipo degenerativo aumentan con el uso de aceite de pescado, comparado con el aceite de soya. Esto indica que su uso no es adecuado en la alimentación de tilapias y la pertinencia de evaluar estos efectos en otros peces de agua dulce.

\section{Conclusiones}

El aceite de soya en las tilapias hibridas (Oreochromis $s p p)$, es utilizado más eficientemente que el aceite de pescado como fuente de lípidos. Los perfiles de ácidos grasos son diferentes, tanto para el aceite de soya como para el aceite de pescado. De esta manera, las fuentes de lípidos de origen vegetal, como el aceite de soya, ricas en ácido linoleico y en menor medida ácido linolenico, inciden sobre el rendimiento productivo positivamente y disminuyen la presentación de hígado graso. Por otra parte, las fuentes de lípidos de origen animal, altas en PUFAS de cadena larga como el aceite de pescado, principalmente ácido docosahexaenoico (DHA) y eicosapentaenoico (EPA), influyen de una manera negativa en el rendimiento productivo, y au-
Tabla 5. Perfil de ácidos grasos en las dietas evaluadas.

\begin{tabular}{lrrrrrr}
\hline \multicolumn{7}{c}{ Porcentaje de acidos grasos en las dietas evaluadas } \\
\hline \multicolumn{1}{c}{ Pufas } & AP $\mathbf{5 \%}$ & AP 9\% & AP $\mathbf{1 3} \%$ & AS $\mathbf{5 \%}$ & AS $\mathbf{9 \%}$ & AS 13\% \\
\hline C8:0 & 0,26 & 0,05 & 0,03 & 0,01 & 0,03 & 0,04 \\
C10:0 & 0,61 & 0,13 & 0,1 & 0,04 & 0,06 & 0,03 \\
C12:0 & 0,75 & 0,27 & 0,24 & 0,05 & 0,05 & 0,03 \\
C14:0 & 11,21 & 10,29 & 10,58 & 0,28 & 0,26 & 0,45 \\
C15:0 & 1,06 & 0,87 & 0,89 & 0,04 & 0,04 & 0,05 \\
C16:0 & 27,39 & 22,86 & 23,32 & 11,2 & 11,18 & 11,31 \\
C16:1 & 10,95 & 12,07 & 12,34 & 0,17 & 0,21 & 0,5 \\
C17:0 & 0,74 & 0,65 & 0,67 & 0,11 & 0,1 & 0,12 \\
C17:1 & 1,01 & 1,36 & 0,16 & 0,05 & 0,05 & 0,05 \\
C18:0 & 5,4 & 4,2 & 4,23 & 4,1 & 4,05 & 4,03 \\
C18:1n-9t & 0,21 & 0,08 & 0,08 & 0,07 & 0,06 & 0,06 \\
C18:1n-9c & 9,72 & 8,36 & 8,16 & 19,85 & 19,84 & 19,45 \\
C18:1n-7 & 3,73 & 3,77 & 3,77 & 2,36 & 2,42 & 2,46 \\
C18:2n-6c & 7,15 & 3,57 & 3,29 & 53,57 & 53,59 & 52,62 \\
C20:0 & 0,27 & 0,23 & 0,22 & 0,28 & 0,28 & 0,28 \\
C18:3n-6 & 0,21 & 0,3 & 0,3 & 0,19 & 0,19 & 0,2 \\
C20:1 & 0,83 & 1,04 & 1,07 & 0,37 & 0,34 & 0,43 \\
C18:3n-3 & 1,35 & 1,53 & 1,6 & 6,8 & 6,58 & 6,81 \\
C20:2 & 1,38 & 2,22 & 2,22 & 0,05 & 0,03 & 0,02 \\
C22:0 & 0,18 & 0,13 & 0,13 & 0,29 & 0,29 & 0,36 \\
C20:3n-6 & 0,1 & 0,15 & 0,15 & 0,02 & 0,02 & 0,02 \\
C22:1n-9 & 0,15 & 0,18 & 0,17 & 0,02 & 0,02 & 0,03 \\
C20:3n-3 & 0,2 & 0,26 & 0,26 & 0 & 0 & 0,02 \\
C23:0 & 0,52 & 0,86 & 1,02 & 0 & 0 & 0,03 \\
C20:5n.3 & 6,85 & 11,76 & 11,94 & 0,03 & 0,1 & 0,32 \\
C22:5n-3 & 1,2 & 2,14 & 2,14 & 0,01 & 0,12 & 0,04 \\
C22:6n-3 & 6,58 & 10,66 & 10,91 & 0,03 & 0,07 & 0,23 \\
\hline Total & 100 & 100 & 100 & 100 & 100 & 100 \\
\hline PUFAS & 25,02 & 32,6 & 32,82 & 60,69 & 60,71 & 60,29 \\
MUFAS & 26,59 & 26,86 & 25,75 & 22,89 & 22,94 & 22,99 \\
SFAS & 48,4 & 40,54 & 41,43 & 16,41 & 16,35 & 16,73 \\
n-3 & 16,18 & 26,36 & 26,83 & 6,86 & 6,88 & 7,42 \\
n-6 & 7,46 & 4,02 & 3,74 & 53,78 & 53,8 & 52,84 \\
n6/n3 & 0,46 & 0,15 & 0,14 & 7,84 & 7,82 & 7,12 \\
\hline
\end{tabular}

mentan la presentación de hígado graso. Se destaca que si bien hay diferencias entre las dos fuentes de lípidos, entre los porcentajes de inclusión evaluados no se observaron estas diferencias. Lo anterior indica que los niveles menores de inclusión (5\%) son igualmente eficientes que los niveles mayores de inclusión (9 y 13\%), siempre y cuando los requerimientos de ácidos grasos esenciales sean suplidos en la dieta.

\section{Agradecimientos}

Los autores agradecen al Instituto de Acuicultura de la Universidad de los Llanos, a la dirección general de investigaciones de la Universidad de los Llanos y a la Universidad Nacional de Colombia por el apoyo logístico y financiero prestado para la realización de este trabajo. 


\section{Referencias}

Aguiar A, Rodrigues D, Pereira L, Braidotti F, Laguila E, Visentainer $\mathrm{V}$. Effect of flaxseed oil in diet on fatty acid composition in the liver of Nile Tilapia (Oreochromis niloticus). Arch Latinoam Nutr. 2007; 57:273-277.

Al-Souti A, Al-Sabahi J, Soussi B, Goddard S. The effects of fish oil-enriched diets on growth, feed conversion and fatty acid content of red hybrid tilapia, Oreochromis sp. Food Chemistry, 2012;133:723-727.

AOAC 1995.Association of Official Analytical Chemists. Official Methods of Analysis 16th. Arlington, Va.

Asdari R, Aliyu-Paiko M, Hasmin R, Ramacha-Dran S. Effects of different dietary lipid sources in the diet for Pangasius hypophthalmus (Sauvage, 1878) juvenile on growth performance, nutrient utilization, body indices and muscle and liver fatty acid composition. Aquac Nutr. 2011;17:44-53

Bandarra N, Rema P, Batista I, Pousao P, Valente L, Batista $S$, Ozorio O. Effects of dietary $n-3 / n-6$ ratio on lipid metabolism of gilthead seabream (Sparus auratus). Eur.J.Lipid.sci.Technol. 2011;113:1332-1341.

Boorman G, Botts S, Bunton T, Fournie J, Harshbarger J, Hawkins W, Hinton D, Jokinen M, Okihiro M, Wolfe M. Diagnostic criteria for degenerative, inflammatory, proliferative nonneoplastic and neoplastic liver lesions in medaka (Oryzias latipes): consensus of a National Toxicology Program Pathology Working Group. Toxicol Pathol, 1997;25:202-10.

Caballero M, Izquierdo M, Kjorsvik E, Fernández A, Rosenlund G. Histological alterations in the liver of sea bream, Sparus aurata, caused by short or long term feeding with vegetable oils. Recovery of normal morphology after feeding fish oil as the sole lipid source. J Fish Dis. 2004;27:531-541.

Caballero MJ, López G, Socorro J, Roo FJ, Izquierdo MS, Férnandez AJ. Combined effect of lipid level and fish meal quality on liver histology of gilthead seabream (Sparus aurata). Aquaculture. 1999;179:277-290.

El-Husseiny O, Abdul-Aziz G, Goda A, Suloma A. Effect of altering linoleic acid and linolenic acid dietary levels and ratios on the performance and tissue fatty acid profiles of Nile tilapia Oreochromis niloticus fry. Aquac Inter. 2010; 18:1105-1119.

Glencross B. Exploring the nutritional demand for essential fatty acids by aquaculture species. Reviews in Aquaculture, $2009 ; 1: 71-124$

Gang J, Jian F, Zhibiao Q. Studies on the Fatty Liver Diseases of Sciaenops ocellatus Caused by Different Ether Extract Levels in Diets. Front. Biol. China, 2006:9-12.

Hardy RW. The nutritional pathology of teleosts. Fish Pathology, Edited by Ronald J Roberts., Chapter 2012;10: 402-424.

Huang C, Huang M, Hou P. Effect of dietary lipids on fatty acid composition and lipid peroxidation in sarcoplasmic reticulum of hybrid tilapia, Oreochromis niloticus $\times O$. aureus. Comparative Biochemistry and Physiology Part B: Biochem Mol Biol. 1998; 120:331-336.
Iregui C, Hernández E, Jiménez A, Pulido A, Rey A, Comas J, Peña C, Rodríguez M. Primer mapa epidemiológico de las lesiones y enfermedades de los peces en Colombia. Universidad Nacional de Colombia. Facultad de Medicina Veterinaria y zootecnia. 2004;1:9-45.

Kanazawa A, Teshima S, Sakamoto M. Requirements of Tilapia zillii for essential fatty acid. Bull Jap Soc Sci Fish. 1980; 46:1353-1356.

Lim C, Yildirim M, Klesius P. Lipid and Fatty Acid Requirements of Tilapias. North American Journal of Aquaculture. $2011 ; 73: 188-193$.

Luna L. 1968. Methods for fats and Lipids. In: Manual of Histologic Staining Methods of the Armed Forces Institute of Pathology, McGraw Hill Books. Chapter 9, 140-152.

Oliva A. Nutrition and health of aquaculture fish. J Fish D. 2012; 35:83-108.

Olsen R, Henderson R, Mcandrew B. The conversion of linoleic acid and linolenic acid to longer chain polyunsaturated fatty acids by Tilapia nilotica (Oreochromis niloticus) in vivo. Fish Physiol Biochem. 1990; 8:261-270.

Rey A. 2002. Caracterización de las enfermedades de la tilapia roja (Oreochromis spp) cultivadas en el departamento del Tolima, sistematización de la información y fisiopatología de la enfermedad septicémica. Universidad Nacional de Colombia-Facultad de Medicina Veterinaria y zootecnia., Tesis de Maestría en Salud y Producción animal, 94

Roberts R. 2012. The aquatic environment. Fish Pathology, Edited by Ronald J Roberts., Chapter 1, 1-16.

Rondon I, Eslava P. Hígado graso en tilapias de cultivo: ¿la punta del iceberg de un problema metabólico asociado a desequilibrio nutricional y de calidad del agua en sistemas de producción? XV Jornada de Acuicultura-Instituto de Acuicultura Universidad de Los Llanos (IALL). 2009;15:70-76.

Sargent J, Henderson R, Tocher D. 1989. The lipids. Fish Nutrition, Edited by John E Halver, Second Edition, 153-218.

Spisni E, Tugnoli M, Ponticelli A, Mordenti T, tomasi V. Hepatic steatosis in artificially fed marine teleosts. J Fish Dis. 1998; $21: 177-184$

Sales J, Glencross B. A meta-analysis of the effects of dietary marine oil replacement with vegetable oils on growth, feed conversion and muscle fatty acid composition of fish species. Aquac Nutr. 2011; 17:e271-e287.

Szabo A, Mezes M, Hancz C, Molnar T, Varga D, Romvari $\mathrm{R}$, Febel $\mathrm{H}$. Incorporation dinamics of dietary vegetable oil fatty acids into the triacylglycerols and phospholipids of tilapia (Oreochromis niloticus) tissues (fillet,liver,visceral fat and gonads). Aquac Nutri. 2011; 17: 132-147.

Takeuchi T, Satoh S, Watanabe T. Requirement of Tilapia nilotica for Essential Fatty Acids. Bulletin of the Japanese Society of Scientific Fisheries. 1983;49:1127-1134.

Teoh C, Turchini G, Wing-Keon N. Erratum to "Genetically improved farmed Nile tilapia and red hybrid tilapia showed differences in fatty acid metabolism when fed diets with added fish oil or a vegetable oil blend". Aquaculture. 2011; 316: 144-154. 
Tocher D. 2003. Metabolism and Functions of Lipids and Fatty Acids in Teleost Fish. Rev Fish Sci. 2003; 11: 107-184.

Tocher D, Agaba M, Hastings N, Bell J, Dick J, Teale A. Nutritional regulation of hepatocyte fatty acid desaturation and polyunsaturated fatty acid composition in zebrafish (Daniorerio) and tilapia (Oreochromis niloticus). Fish Physiol Biochem. 2001a;24: 309-320.
Tocher D, Bell J, Macglauchlin P, Mcghee F, Dick J. 2001b Hepatocyte fatty acid desaturation and polyunsaturated fatty acid composition of liver in salmonids: effects of dietary vegetable oil. Comparative Biochemistry and Physiology part B, 257-270.

Wolf J, Wolfe M. A Brief Overview of Nonneoplastic Hepatic Toxicity in Fish. Toxicol Pathol. 2005; 33:75-85. 